\title{
Médiévales
}

Langues, Textes, Histoire

50 | printemps 2006

Sociétés nordiques en politique ( $\mathrm{XII}{ }^{\mathrm{e}}-\mathrm{XV}^{\mathrm{e}}$ siècles)

\section{« Aïmanz »: un chapitre de l'« encyclopédie lyrique » de Gautier d'Épinal}

"Aïmanz »: a Chapter of Gautier D'Épinal's « Lyrical Encyclopedia ».

\section{Germana Schiassi}

\section{(2) OpenEdition}

12 Journals

\section{Édition électronique}

URL : https://journals.openedition.org/medievales/1391

DOI : 10.4000/medievales. 1391

ISSN : $1777-5892$

Éditeur

Presses universitaires de Vincennes

\section{Édition imprimée}

Date de publication : 1 juin 2006

Pagination : 155-167

ISBN : 2-84292-186-0

ISSN : 0751-2708

\section{Référence électronique}

Germana Schiassi, « « Aïmanz » : un chapitre de l'« encyclopédie lyrique » de Gautier d'Épinal », Médiévales [En ligne], 50 I printemps 2006, mis en ligne le 15 septembre 2008, consulté le 22 avril 2022. URL : http://journals.openedition.org/medievales/1391 ; DOI : https://doi.org/10.4000/ medievales. 1391

Ce document a été généré automatiquement le 22 avril 2022.

Tous droits réservés 


\title{
« Aïmanz » : un chapitre de l'« encyclopédie lyrique » de Gautier d'Épinal
}

« Aïmanz »: a Chapter of Gautier D'Épinal's «Lyrical Encyclopedia ».

\author{
Germana Schiassi
}

1 La chanson Tout autresi com l'aïmanz deçoit ${ }^{1}$, qui fait l'objet du présent article, est attribuée à Gautier d'Épinal, chevalier lorrain dont la production littéraire date du XIII siècle. Attesté dans plusieurs documents entre 1232 et 1270, Gautier d'Épinal est l'auteur d'une quinzaine de chansons d'argument courtois, qui ont pour destinataires ses nombreux protecteurs, notamment les comtes de Bar (Henri II et Thibaut II) et les comtes de Vaudémont (Hugues II et Hugues III) ${ }^{2}$.

2 Beaucoup d'indices font penser que, en son temps, le trouvère avait joui d'une certaine popularité, ses chansons étant l'objet de plusieurs contrafacta pieux ou bien se trouvant parmi les nombreuses insertions lyriques dans le roman Méliacin de Girart d'Amiens ${ }^{3}$.

Cela n'a pourtant pas empêché que l'œuvre poétique de Gautier ait été sous-estimée, voire ignorée par la critique moderne et contemporaine ${ }^{4}: c^{1}$ est sans doute le jugement sévère formulé en 1902 par Alfred Jeanroy ${ }^{5}$, au lendemain de la parution de l'édition de Gautier signée par Ugo Lindelöf et Axel Wallensköld ${ }^{6}$, qui a décidé de façon négative de la fortune du trouvère à notre époque.

4 L'exigence d'une nouvelle édition de Gautier d'épinal est donc pressante, d'autant plus que, contrairement à ce qu'affirmait Jeanroy, certaines chansons de son corpus se signalent par la richesse et l'originalité de l'invention poétique, et méritent d'être comptées au nombre des témoignages les plus illustres de la production lyrique européenne au XIII ${ }^{\mathrm{e}}$ siècle.

5 En particulier, la chanson RS. 1840 a retenu notre attention: nous en donnons cidessous le texte dans l'édition Lindelöf-Wallensköld ${ }^{7}$ : 
Tot autresi com l'aïmanz deçoit

L'aguillete par force de vertu,

A ma dame tot le mont retenu

Qui sa biauté conoist et aperçoit,

Et je sui cil qui sor toz la covoit ; 5

Et se n'i truis ne merci ne raison

Par quoi puisse venir a garison,

Bien ait l'amors qui por mal ne recroit !

II

Si com l'arbres qui encontre le froit

Se tient de flors et de sa fueille nu 10

Ai je mon sens oblié et perdu

Vers ma dame, quant plus mestier m'avroit.

Ahi, biaus Deus ! s'ele bien conoissoit

La grant amor, la bone entention

Dont li sospir vienent a tel foison, 15

Ja fins amis a joie ne faudroit.

III

Ne voudroie joïr de rien qui soit

Se ce par li ne m'estoit avenu,

Si com la lune a son veoir perdu,

Quant la clarté del soleil ne reçoit. 20

He, franche riens ! s'a nul jor avenoit

Qu'eüssiez ja ne merci ne pardon

D'ami verai loial sans traïson,

Por Deu, gardez al mains que ce seroit !

IV

A semblance d'ome qui adés boit 25

Ne ja nul jor n'avra assez beü,

M'a fait ma dame et ydropique et mu,

Que ne li os descovrir rien qui soit.

Quant plus l'esgart et je plus la covoit,

Et quant je n'os remirer sa façon 30

Li ueil del cuer remirent a bandon

Et pensent ce que nus hom ne verroit.

$\mathrm{V}$

Tout autresi com de l'ombre qu'on voit

Que ce n'iert ja ne senti ne tenu,

M'a ma dame sans plaie el cors feru ; 35

Mais je ne sai dont ce vient ne que doit,

Fors d'un regart que je vi qu'ele avoit ;

Mais or le truis si cruël et felon

K'ainçois l'avroit cent mile foiz uns hom

Resgardee, qu'ele lui n'en avroit. 40

VI

Chançons, di moi a mon seignor Huon :

Si vain sohait et si espoir breton

M'ont deceü ; car ce est a bon droit.

Datée par Holger Petersen Dyggve des années 30-40 du XIII ${ }^{\mathrm{e}}$ siècle ${ }^{8}$, cette chanson se déroule en cinq strophes dont chacune contient une image tirée du monde naturel. Pourtant, la nature n'est pas ici un prétexte pour l'invention poétique, ni le simple décor d'une narration. Dans chaque strophe, l'auteur décrit systématiquement les effets de l'influence amoureuse de la dame sur l'amant par le biais d'une comparaison avec un phénomène naturel toujours différent : le magnétisme, la chute des feuilles en hiver, l'éclipse, l'hydropisie, l'ombre. Ces phénomènes touchant aux domaines les plus 
divers, le poète utilise pour chacun d'eux un lexique scientifique pertinent et rigoureux. Pour la variété d'arguments et pour la précision du vocabulaire, on peut considérer chaque strophe comme un chapitre d'une petite encyclopédie lyrique, dans laquelle imago mundi et imago amoris se reflètent et se superposent.

Dans les pages qui suivent, nous concentrerons notre attention sur le premier « chapitre » de cette petite encyclopédie amoureuse, celui qui s'ouvre avec l'image de l'aimant : s'il est vrai que Gautier n'a pas été le premier à décrire le charme de la dame à travers cette pierre, néanmoins le choix des mots, des expressions et des renvois à d'autres passages à l'intérieur de la même chanson témoignent du renouvellement de la lyrique du XIII ${ }^{e}$ siècle dans ses figures au contact des savoirs scientifiques. D'abord, nous tâcherons de parcourir brièvement l'évolution du mot aimant et de son acception; ensuite, nous essayerons de repérer les sources littéraires et scientifiques dans lesquelles Gautier d'Épinal aurait puisé, élaboré et renouvelé son image. Nous démontrerons, en dernière étape, que son apport n'est pas isolé dans le cadre de la production lyrique contemporaine, mais qu'il trouve des correspondances très ponctuelles dans le milieu le plus «expérimental» de l'époque, celui de l'«École» poétique sicilienne de Frédéric II.

Histoire d'une équivoque

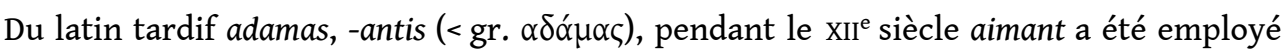
pour désigner tantôt le " diamant », tantôt l'« aimant ». Les dictionnaires philologiques et étymologiques se bornent à enregistrer la double acception du terme ${ }^{9}$. Il est intéressant de noter que, si la confusion au niveau lexical se produit à partir des langues vulgaires, une superposition partielle au niveau des propriétés des deux pierres est attestée depuis l'Antiquité. Étant donné que la catégorie de pierres appelée adamas par les auteurs anciens comprenait aussi des métaux ${ }^{10}$, Pline l'Ancien dans le livre XXXVII de sa Naturalis Historia attribuait au diamant des vertus magnétiques ${ }^{11}$. Lorsque Isidore de Séville s'occupe du diamant dans le livre XVI des Etymologiae, il reprend la tradition de Pline ${ }^{12}$, et représente à son tour la source principale des vers consacrés au diamant dans De lapidibus de Marbode, évêque de Rennes :

Omnibus [adamantibus] aequa tamen vis est adducere ferrum ; Quod facit et magnes absente potens adamante : Nam praesens adamas magneti, quod rapit, aufert.

[La vertu propre à tous les diamants est celle d'attirer le fer : l'aimant aussi fait cela, il en devient capable en l'absence du diamant. Car lorsque le diamant est présent, il arrache à l'aimant ce qu'il a attiré] ${ }^{13}$.

En revanche, en ce qui concerne l'aimant, Marbode utilise entièrement une tradition d'ascendance aristotélicienne, celle du traité de Damigéron : l'original grec étant perdu, il ne nous reste que la traduction latine ${ }^{14}$ :

Est autem de colore ferrugineus, magnas habens in se virtutes, veluti spiritum in attrahendo sibi ferrum et retinendo. Inuenientes autem eum colchi, dicunt hoc lapide usum esse in magica actione [...] Vires habet preterea purgatorias. et ideo ydropicis cum mulsa datus [...] Tritus et combustionibus super aspersus eas sanat. [Il est de la couleur du fer, et il possède de grandes vertus, comme le pouvoir d'attirer vers soi le fer, et de le retenir. Les Colchidiens qui le repèrent prétendent que cette pierre est utilisée pour des pratiques de magie. De plus, la pierre a des propriétés purgatives, et c'est pour cela qu'elle est administrée aux hydropiques, avec du vin miellé. Pilé et aspergé sur les brûlures, il les guérit] ${ }^{15}$.

10 De ce dernier ouvrage Marbode tire les propriétés médicales relatives à l'aimant : 
Gratia praestantur simul, et suadela per ipsum, sermonisque decor, disceptandique facultas. Cum mulso datus, hydropem purganda resolvit. Et combusturas super aspersus medicatur.

[Grâce à l'aimant, le pouvoir de la persuasion est assuré, ainsi que la pertinence de l'expression et la force de l'argumentation. Administré avec du vin miellé à l'usage purgatif, l'aimant libère l'hydropique, et aspergé sur les brûlures, les soigne] ${ }^{16}$.

Les adaptations successives en français de l'ouvrage de Marbode ne s'éloignent généralement pas de leur source. Pourtant, dans la Première version en vers en anglonormand, le traducteur, en parlant des propriétés de l'aimant (appelé « Magnete »), dit que « Fer resemble e si le trait Ausi cume l'aïmant fait » (v. 455-6) ${ }^{17}$, ce dernier vers étant absent dans le chapitre de Damigéron. Le traducteur serait-il cultivé au point de ressentir l'exigence d'ajouter cette spécification pour rattraper la tradition plinienne ${ }^{18}$ ? Ou, plus vraisemblablement, aurait-il pris conscience des possibles ambiguïtés engendrées par l'identité lexicale du terme, qui se produisent au moins jusqu'au milieu du XIII ${ }^{e}$ siècle ${ }^{19}$ ? Cela apparaît évident dans la Première version en vers au niveau des variantes formelles : dans le ms. A (Paris, BnF lat. 14470), daté par Studer de la deuxième moitié du XII ${ }^{\mathrm{e}}$ siècle ou du début du XIII ${ }^{\mathrm{e}}$ siècle ${ }^{20}$, « adamas » est traduit par aïmant. Par contre, les manuscrits qui ont été copiés, d'après Studer, vers la fin du XIII ${ }^{\mathrm{e}}$ siècle écrivent dyamant/diamant/adamas: cela veut dire qu'à cette époque, la distinction entre «aymant » et «diamant» est plus nettement fixée dans la langue. D'ailleurs, le mot « magnete », utilisé couramment dans les lapidaires en vulgaire, n'est plus attesté depuis la fin du $\mathrm{xIII}^{\mathrm{e}}$ siècle ${ }^{21}$ : à titre d'exemple, lorsqu'en 1371 Jean Corbechon traduit en français le XVI livre du De Proprietatibus rerum de Barthélemy l'Anglais ${ }^{22}$, il traduit "magnes » par aimant, mais, tout en essayant de garder l'ordre alphabétique du classement des pierres dans l'original latin, il ne change pas de place à l'aimant, qui se trouve sous la lettre « $\mathrm{m} »{ }^{23}$.

12 Vers 1288, c'est le célèbre médecin Simon de Gênes qui s'efforce de résoudre l'équivoque lexicale et, en même temps, de combattre les fausses (d'après son opinion) croyances liées à cette pierre. Dans son lexique intitulé Clavis sanationis (édition de Padoue 1474), à l'entrée « adamas », il écrit :

Adamas, lapis durissimus, inter preciosas gemmas computatus, sex angulos habens, ferruginei coloris, quem multi mentiti sunt non posse frangi nisi cum yrcino sanguine, aut plumbo, quod aurifabri experti negant. Aliquando adamas magnes vocatur [...]. Lapis magnes, qui ferrum trahit, multi adamantem putaverunt falso.

[Adamas, pierre très dure, au nombre des pierres précieuses, avec six angles, de la couleur du fer, dont plusieurs ont affirmé faussement qu'elle ne pourrait être brisée que par le sang de bouc, ou par le plomb - ce que les orfèvres experts nient. Parfois elle est appelée "aimant». Plusieurs ont faussement pris la pierre aimant, qui attire le fer, pour le diamant] ${ }^{24}$.

13 Dans les témoignages littéraires, l'ambiguïté lexicale entre les deux pierres est largement attestée, au moins jusqu'au troisième quart $\mathrm{du} \mathrm{xIII}^{\mathrm{e}}$ siècle. Parmi les nombreux exemples recueillis par Tobler-Lommatzsch et par Ziltener, celui du Roman de la Rose nous semble assez exhaustif : [diamant] Guill. Lorr., 3842 : «La pierre est de roche naïve De quoi l'en fist le fondement, Si est dure come aïmant"; J. de Meun, v. 16333 : «Perceroit pierre d'aiment »; [aimant] Guill. Lorr. 1158 : « Car trestot en autel maniere Con la pierre de l'aiment Trait a soi le fer soutilment Ausi atrait le cuer des genz Li ors qu'en done et li argenz »; J. de Meun, v. 19340 : «Plus se vuet [Amour] vers mes [Nature] euvres traire Que ne fait fer vers aimant ${ }^{25}$.

Les sources de Gautier d'Épinal : entre tradition et innovation 

largement utilisée par les troubadours ${ }^{26}$ et ensuite par les poètes de «l'École sicilienne ${ }^{27}$. D'ailleurs, l'utilisation de l'aimant, ainsi que d'autres pierres précieuses, n'est pas un cas isolé dans sa production ${ }^{28}$. tradition que l'aimant est utilisé pour guérir l'hydropisie et pour augmenter le charme de celui qui le porte, en lui conférant le don de l'éloquence ${ }^{29}$; cela nous conduirait à penser que Gautier avait une connaissance directe d'un de ces textes : nous ne serions pas en mesure d'expliquer autrement la raison pour laquelle le poète, dans la même chanson, utilise l'image de l'aimant et dit que la dame l'a rendu "ydropique et mu ", cette association ne se trouvant pas ailleurs, à notre connaissance, dans la lyrique en ancien français, ni provençale ni italienne. Les deux motifs sont liés aussi par celui de la maladie : la dame, en tant qu'objet de « convoitise » (v. 5, 29), est la cause de la maladie (v. 7, 27-29) du poète, et, en tant qu'« aimant », le seul antidote qui puisse lui donner la guérison (v. 7).

On ne peut pas non plus exclure que Gautier ait pris connaissance de nombreuses expériences scientifiques sur le magnétisme, conduites au cours du xiII siècle en Europe, qui avaient d'ailleurs sans doute contribué au développement de ce motif dans la poésie lyrique contemporaine ${ }^{30}$. Le choix des mots est aussi révélateur de la tentative de plier à l'image poétique le phénomène naturel observé et évalué de façon strictement scientifique. Lorsqu'il parle de l'aimant (mais le discours serait valable pour chacune des images présentes dans la chanson), Gautier ne dit pas simplement qu'il «attire» le fer, mais il utilise le verbe «dechevoir ». Parmi ses significations, le dictionnaire Tobler-Lommatzsch signale celle de «charmer, attraper par enchantements, ensorceler ${ }^{31}$ ». Cette acception pourrait être confirmée par le fait que tous les lapidaires soulignent que l'aimant était la pierre préférée des magiciens: prenons à titre d'exemple la Premier version, vv. 457 svv. : "Dendor l'ama mult durement, Qi l'usot a enchantement. Circél'usa e l'ot mult chere, Cele merveilose sorcere ${ }^{32}$ ".

Gautier introduit encore un élément, celui de l'« aguillete », qui ne se trouve pas dans les lapidaires que nous avons analysés. Il paraît évident que l'auteur fait plutôt référence à l'expérience de l'aiguille aimantée, en particulier celle de la boussole marine, qui d'ailleurs avait été déjà mentionnée chez plusieurs auteurs, dès la fin du $\mathrm{XII}^{\mathrm{e}}$ siècle. On la retrouve, par exemple, dans l'encyclopédie scientifique De naturis rerum d'Alexandre Neckham qui écrit :

Nautae etiam mare legentes, cum beneficium claritatis Solis, in tempore nubilo, non sentiunt, aut etiam cum caligine noctuarum tenebrarum mundus obvolvitur, et ignorant in quem mundi cardinem prora tendat, acum super magnetem ponunt, quae circulariter circumvolvitur usque dum, eius motum cessante, cuspis ipsius septemtrionalis plagam respiciat.

[Les marins qui parcourent la mer, lorsqu'ils ne bénéficient pas de la lumière du soleil à cause du temps nuageux ou encore, lorsque le monde est enveloppé par les ténèbres épaisses de la nuit, de sorte qu'ils ignorent vers lequel des points cardinaux du monde la proue tend, ils posent une aiguille sur un aimant. L'aimant tourne en rond, jusqu'à ce que, une fois qu'il a cessé de tourner, sa pointe regarde en direction du Nord] ${ }^{33}$.

Encore, dans le Speculum Majus de Vincent de Beauvais :

[Magnes] stellamque maris indicem itineris, inter obscuras nebulas per diem vel noctem nautis prodit. Cum enim vias suas ad portum dirigere nesciunt, accipiunt acum, et acumine eius ad adamantem lapide fricato per transversum in festura 
parva figunt et vasi pleno aqua immittunt. Tunc adamantem vasi circumducunt et mox, secundum motum eius, sequitur in circuitu, cacumen acus : rotatum ergo perinde citius, lapidem subito retrahunt; moxque cacumen acus, avulso ductore, contra stellam aciem dirigit, statimque subsistit, nec per puncto movetur; et nautae secundum demonstrationem factam, ad portum vias dirigunt.

[L'aimant révèle aussi aux marins l'astre de la mer, qui indique la route, parmi les nuages obscurs pendant la journée ou pendant la nuit. Car, quand ils ne savent pas revenir au port, ils prennent une aiguille, la fixent dans une petite fente dans la pierre de l'aimant, qui a été frottée obliquement, et ils posent tout cela dans un vase rempli d'eau. Ensuite, ils font tourner en rond l'aimant dans le vase, et aussitôt, selon le mouvement de celui-ci, l'extrémité de l'aiguille en suit la rotation ; lorsque sa vitesse augmente de la même manière, ils retirent soudainement la pierre. Alors, l'extrémité de l'aiguille, libérée de son guide, dresse sa pointe en direction de l'étoile et s'arrête, sans bouger, et les marins, après cette démonstration, se dirigent vers le port] ${ }^{34}$.

Dernier exemple, cette fois en vulgaire, tiré de la Bible de Guiot de Provins, « achevée sans doute au début de $1206^{35} »$ :

De notre pere l'Apostoile

Vouisisse qu'il semblast l'estoile

Qui ne se meut : moult bien la voient...

Ils l'appellent la Tresmontaine...

Un art font, qui mentir ne puet,

par la vertu de la mannete

une pierre laide et brunete

ou li fers volontiers se joint

Ont si esgardent le droit point

Puis qu'une aguille l'ait touché

Et en un festu l'ont fiché...

Quant la mer est obscure et brune

Qu'on ne voit estoile ne lune

Donc font l'aguille allumer

Puis n'ont il garde d'esgarer

Contre l'estoile va la pointe

Par ce sont li marinier cointe

De la droite voie tenir ${ }^{36}$.

Dans cette perspective, la comparaison avec la dame qui a tot le mont retenu (v. 3) est d'autant plus pertinente que, comme l'aiguille aimantée reconnaît et indique l'étoile polaire aux marins, les gens sous les effets du charme magnétique de la dame aperçoivent et reconnaissent sa beauté. Le choix du verbe "dechevoir» est donc révélateur de l'effort du poète pour exprimer une expérience à la fois scientifiquement évaluée, et pourtant encore chargée de mystère et de merveille aux yeux des observateurs ${ }^{37}$. Ce n'est pas par hasard que Pierre de Maricourt, quelques dizaines d'années plus tard, dans le premier chapitre de son Epistola de magnete ad Sygerum de Foucaucourt Militem écrite à Lucera en 1269, précise que les expérimentations dont il va parler :

(...) de quibus quesivisti, appellem manifesta, erunt tamen inextimabilia, et vulgo quasi illusiones et fantasmata; et ideo, quo ad vulgum, secreta sunt: astrologis autem et naturalibus satis erunt manifesta, et ipsis erunt solatium, et provectis viatoribus non modici erunt iuvamenti.

[Les expérimentations au sujet desquelles tu m'as interrogé, je les appellerai évidentes, cependant, pour le peuple, elles seront impossibles à juger, et presque des illusions ou des spectres; c'est pour cela qu'elles demeurent secrètes au peuple : mais elles seront assez évidentes pour les astrologues et les naturalistes, et pour 
ceux-ci elles seront une ressource, et les voyageurs expérimentés en tireront beaucoup de bénéfices] ${ }^{38}$.

Correspondances dans la lyrique du XIII ${ }^{\mathrm{e}}$ siècle

Restons en Italie et revenons à l'« École sicilienne », qui naît et se développe au sein de la magna curia de Frédéric II de Hohenstaufen. L'empereur, qui règne de 1220 à 1250, s'intéresse à la philosophie naturelle et à l'astrologie; il encourage l'activité scientifique et les traductions de textes grecs et arabes, il est, lui-même, avec probabilité, l'auteur du fameux traité de fauconnerie De arte venandi cum avibus. Par conséquent, les poètes dits «Siciliens ", souvent des fonctionnaires de la cour, reflètent et partagent l'unicité du milieu culturel dont ils font partie : nous remarquerons en particulier la richesse de métaphores et d'images tirées de la philosophie naturelle.

Si la datation de Holger Petersen Dyggve est exacte, RS. 1840 serait donc contemporaine de certains exemplaires de la production poétique de l'École sicilienne, qui présentent une visible affinité de structures et thèmes: nous songeons, par exemple, à Giacomo da Lentini, Madonna dir vo voglio, qui est à son tour une traduction de la chanson A vos midontç, voill rettrair'en cantan de Folquet de Marseille; ou encore, à Guido delle Colonne, Ancor che l'aigua per lo foco lassi, qui d'ailleurs utilise et développe largement l'image de l'aimant ${ }^{39}$ :

La calamita contano i saccenti

Che trare non poria

Lo ferro per maestria,

se no che l'aire in mezzo le 'l consenti.

Ancor che calamita petra sia,

L'altre petre neenti

Non son cusì potenti

A traier perché non hano bailia.

Così, madonna mia,

l'Amor s'è apperceputo

che non m'avria potuto

traer' a sé se non fusse per voi ;

e sì son donne assai,

ma no nulla per cui

eo mi movesse mai,

se non per voi, piagente,

in cui è fermamente

la forza e la vertuti ${ }^{40}$.

L'exemple qui suit est encore plus frappant : la chanson Lo gran valore e lo presio amoroso de Mazzeo di Ricco, attesté entre 1252 et 1260, présente le même emploi de l'image que chez notre auteur :

\author{
E serete sicura \\ Che la vostra bellezze mi ci 'nvita \\ Per forza, come fa la calamita \\ Quando l'aguglia tira per natura ${ }^{41}$.
}

24 La recherche est ouverte : nous ne prétendons pas établir une source textuelle à la base de Gautier d'Épinal et des Siciliens. La lecture de la strophe du RS. 1840 se borne à montrer que, si d'un côté le genre lyrique devient au cours du XIII ${ }^{\mathrm{e}}$ siècle de plus en plus cristallisé et clos au niveau des thèmes et des structures ${ }^{42}$, de l'autre il se renouvelle et s'enrichit au niveau de l'ornatum: grâce à la diffusion des ouvrages scientifiques souvent recueillis en encyclopédies, la poésie lyrique se fait à son tour imago mundi, 
dont la chanson de Gautier nous parait un exemple particulièrement réussi dans l'union de la rigueur scientifique et de l'inventio poétique.

\section{NOTES}

1. La chanson sera mentionnée par la suite avec le sigle RS. 1840, telle qu'elle est indiquée dans la bibliographie de G. RAYNAUD - H. SPANKE, G. Raynauds Bibliographie des altfranzösischen Liedes, neu bearbeitet und ergänzt von H. Spanke, Leyde, 1955.

2. Pour les questions relatives à la chronologie de l'auteur et de ses chansons voir H. Petersen DyGgVE, « Personnages historiques figurant dans la poésie lyrique française des XII et XIII siècles. II, Gautier d'Épinal », Neuphilologische Mitteilungen, XXXVI, 1935, p. 19-29.

3. Voir A. SALY, Girart d'Amiens, Meliacin ou le cheval de fust, Aix-en-Provence, 1990 (Senefiance, 27).

4. Par exemple, l'anthologie très connue de S. N. ROSENBERG et H. TISCHLER, Chansons des trouvères. Chanter m'estuet, Paris, 1995, ne cite Gautier que dans le commentaire d'une chanson de croisade, éditée comme anonyme, Jherusalem, grant damage me fais. La paternité de notre auteur est, en effet, très douteuse : il est étonnant de remarquer qu'aucune des 15 chansons dont l'attribution à Gautier est sûre n'ait été accueillie dans une anthologie qui en compte 217.

5. A. JeAnRoY, « Les chansons de Gautier d'Épinal », Romania, XXXI, 1902, p. 436-9. Voir p. 439, où Jeanroy remarque « la banalité lamentable de ses idées et l'embarras de son style ».

6. U. LINDELÖF et A. WALLENSKÖLD, « Les Chansons de Gautier d'Épinal », Mémoires de la Société Néophilologique à Helsingfors, 3, 1901, p. 205-318.

7. ID., p. 293-295. Un nouveau texte critique de RS. 1840 sera établi dans le cadre d'une nouvelle édition de Gautier d'Épinal - Thèse de doctorat en cours, sous la direction des Professeurs M. L. Formisano (Université de Bologne) et J. Cerquiglini-Toulet (Université de Paris IV-Sorbonne).

8. Cf. Petersen DygGVe, art. cit., p. 26 : « La chanson XIII (R. 1840)... porte comme destinataire mon seignor Huon. Nous pensons qu'on pourra avec une grande vraisemblance y voir ou Hugues II, comte de Vaudémont de 1190 à 1235 ou 1236, ou bien Hugues III, son successeur, comte jusqu'à 1246 , ce qui donnerait pour la composition de la chanson une date antérieure à 1246 ».

9. Cf. F. GODEFROY, Dictionnaire de l'ancienne langue française, 10 vol., Paris, 1881-1902, VIII, compl., p. $59: 1$. « minerai de fer oxydé qui a la propriété d'attirer le fer » [En Ynde croist li aemans (Gauth. de Mes., Ym. du monde, BnF, fr. 2021, fo 103ra)] ; 2. « Diamant, pierre dure comme le diamant ». - cf. A. TOBLER, E. LOMMATzsch, Altfranzösisches Wörterbude, Wiesbaden, 1925-1965, I, p. 240-41. - cf. DU CANGE, Glossarium Mediae et Infimae latinitatis, Niort, 1840, I, p. 69 : « Videtur esse Magnes, Gall. Aimant. Vox Graecae originis. At vero nostris olim Adamas prius dicebatur Aimant, quam vocaretur Diamant ; quae ultraque vox a verbo Adamas origine habet ». - cf. FEW, band I-1, p. 132-133 : « Lat. 
ADAMAS, -antis hat die Bedeutungen « harter stahl » und « diamant ». Apr. aziman weist darauf hin, dass es $\mathrm{zu}^{*}$ adimas dissimiliert worden ist. Das ursprüngliche ADAMAs liegt vor in amail. adamanta « diamant ", siz. damanti RLomb 40, 1146, Novara (siz.) domandi MLomb 21, 269, kat. azamant « magnet ». Aus * adimante die Formen unter I1 [ aimant »]. Die Formen unter 2 [ diamant »] gehen zurück auf ein spätlt. diamas (4.Jh., Ianz 23, 79), das allerdings als Name eines Pferdes belegt ist ; eine solche Namengebung ist sehr wohl möglich. Die Form diamas erklärt sich wohl durch Metathese von adi- unter dem einfluss der vielen griechischen Wörter, die mit diabeginnen, wie diaphanes usw. Unter II [Afr. Mfr. Adamas m. « diamant »] vereinzelte Entlehnungen aus lt. Adamas, -antis ».

10. Pour le problème de l'acception du mot « diamant » dans la littérature scientifique tardo-ancienne et médiévale, voir M. E. HERRERA, « La historia del “diamante” desde Plinio a Bartolomé el Inglés ", dans Comprendre et maîtriser la nature au Moyen Âge. Mélanges d'histoire des sciences offerts à Guy Beaujouan, Genève, 1994, p. 139-153.

11. Pline L'ANCIEN, Histoire naturelle, livre XXXVII, E. DE SAINT-DENIS éd., Paris, 1972, par. 61 : « Adamas dissidet cum magnete in tantum, ut iuxta positus ferrum non patiatur abstrahi aut, si admotus magnes adprehenderit, rapiat atque auferat ».

12. W. M. LINDSAY éd., Isidori Hispalensis Episcopi Etymologiarum sive originum libri XX, Vol. II, Oxford, 1911, XVI, 13, 3 : « Hic [adamas] autem dissidet cum magnete lapide in tantum, ut juxta positus ferrum non patiatur abstrahi, magneti autem si admotus, magnes comprehenderit, rapiat atque auferat. Fertur quoque in electri similitudine venena deprehendere, metus vanos expellere, maleficiis resistere artibus ». - cf. P. STUDER et J. EVANS, Anglo-Norman Lapidaries, Paris, 1924 (réimpression Genève, 1976), 297, n. 42-46.

13. J. RIDDLE, Marbode of Rennes. De lapidibus, considered as a medical treatise, Wiesbaden, 1977, p. 36, par. 40. La croyance selon laquelle la pierre « adamas » arracherait le fer à la pierre « aimant » se retrouve aussi, entre autres, dans le Speculum Majus de Vincent de Beauvais : « Adamantis duo sunt genera, unum in Indiae partibus invenitur inter rupes chrystallinas... hic a quibusdam Diadamas dicitur... ferrum autem hic aufert magneti ", (Biblioteca Mundi Vincentii Burgundi Speculum quadruplex, Douai, 1624, t. I, lib. VIII, C XL).

14. Sur la question des sources du lapidaire, cf. RIDDLE, op. cit., p. 8-21; voir aussi l'article de S. BAGGIO, «Censure Lapidarie », Medioevo romanzo, XI/2, 1986, p. 207-228, en particulier p. 210-214 et R. HALLEUX, « Damigéron, Evax et Marbode : l'héritage alexandrin dans les lapidaires médiévaux », Studi medievali, 15/1, 1974, p. 327-347. 15. J. EvAns, Magical Jewels of the Middle Ages and the Renaissance particularly in England, Oxford, 1922, p. 195 sq. (Appendix A), cap. XXXIV.

16. RIDDLE, op. cit., p. 58, par. 305-306.

17. STUDER, op. cit., p. 46. Les lapidaires successifs présentent la même glose : cf.

L. PANNIER, Les Lapidaires français du Moyen Âge, des XII ${ }^{e}$, XIV et XIV siècles, Paris, 1882 (réimpression Genève, 1973), p. 101 (Lapidaire de Modène), 679 : « Par force le fer a soi trait Ausi com li aïmans fait »; p. 131 (Lapidaire de Berne), 735 : « La pierre et le fer ele atraist Ainsi com li aymant fait ».

18. Lorsque Damigéron parle du diamant, il n'en mentionne aucune propriété magnétique.

19. STUDER, op. cit., 305, n. 46 : « In French the confusion between adamas and magnet is constant, aimant being used for both ». 
20. Ibid., p. 1.

21. Cf. FEW VI-1, 46. Le terme réapparaît au XVII ${ }^{\mathrm{e}}$ siècle (« boiste magnetique ») pour désigner la boussole.

22. JEAN CORBECHON, Le livre des proprietés des choses, Paris, BnF, fr. 16993, fo $225 \mathrm{~d}$ : Le $x v i$ livre qui fait mencion des pierres des couleurs et des metaulx.

23. Ibid., fo $234 b$.

24. T. BERTELLI, «Sulla epistola di Pietro Peregrino di Maricourt e sopra alcuni trovati e teorie magnetiche del secolo XIII. Memoria seconda », Bollettino di bibliografia e di storia delle scienze matematiche e fisiche, Rome, 1868, p. 107 sq.

25. A. TOBLERE, E. LOMMATZSCH, op. cit., I, 240-41; W. ZILTENER, Repertorium der Gleichnisse und bildhaften Vergleiche der okzitanischen und der französischen Versliteratur des Mittelalters, fasc. 1, Literaturverzeichnisse. Unbelebte Natur, Bern, 1972, p. 78-80.

26. Cf. F. RAYNOUARD, Lexique Roman ou dictionnaire de la langue des troubadours, 6 vol., Paris, 1838-1844, II, 162-63 ; ZILTENER, op. cit., 79 : Bernart de Ventadorn, P.-C. 70, 26, VI, 40 « E si m sui de midons lonhans, Vas se m tira com azimans La bela cui Deus defenda »; Folquet de Marselha, P.-C. 155, 20, II 16 (XVII) : " Q'eissamens cum l'azimans Tira 1 fer e 1 fai levar, Fazi'el [Barral] mains cors dreissar Vas pretz, forssatz e pesans »; Aimeric de Peguilhan, P.-C. 10, 12, IV 25 : « A ley del fer que va ses tirador Vas l'aziman que 1 tira vas si gen, Amors, que m sap tirar ses tiramen, Mas tirat m'a sevals per la melhor »; ID. P.-C. 10, 24, I 1 : « Yssamen cum l'äymans Tira l fer e 1 trai vas se, Tir'Amors mon cor ancse, Eu'es forser e mais tirans ».

27. Cf. C. Segre, C. Ossola, Antologia della poesia italiana. Il Duecento, Turin, 1997, p. 47 sq. : [Tenzone con Jacopo Mostacci e Pier della Vigna], vv. 9-11 « Per la vertute de la calamita Como lo ferro atira no si vede Ma sì lo tira signorevolmente ».

28. Cf. RS. 199 où le poète s'adresse à la dame en l'appelant Aimanz fins et verais $\left(\mathrm{v}^{\mathrm{o}} 1\right)$; cf. encore RS. 199, 42-3 : «Et de rubis li balais N'a de biauté nule igance »; RS. 1059, 34-5 : «En la face que je vi, C'est fins rubis et cristaus ».

29. Cf. supra, p. 2 ; L. PANNIER, Les Lapidaires français du Moyen Âge, op. cit., p. 51 (Première version), 488 ; p. 131-2 (Lapidaire de Berne), 781 : « Gracious et amonestant Fait son seignour et bien estant, Del bel parler grace li donne Et d'acoitance bele et bone. La poudre ou most garist et sainne L'idropique, bien li est sainne »; p. 103 (Lapidaire de Modène), 739 : «Sage le fait venir en plait Et belement parler le fait. Li piere est de grant segnerie ; molt est buene por faire aïe, A boire avuec eve molete A ydropikes li manete ». L'association est présente aussi dans le chapitre sur l'aimant du Liber de proprietatibus rerum de Barthélemy l'Anglais : Paris, BnF, ms. lat. 16099, fo 136c, lignes 2-3 : « [Magnes] auget gratiam et decorem in sermone Item $\mathrm{cu}[\mathrm{m}]$ mulsa curat idropisim et splenem.allopitiam et arsuram ».

30. Il faut préciser que les expériences sur le magnétisme ne sont attestées que tardivement dans le XIII ${ }^{\mathrm{e}}$ siècle. À ce propos, voir D. JACQUART, « L'observation dans les sciences de la nature au Moyen Âge : limites et possibilités », Micrologus, 4, 1996, p. 55-75.

31. A. TOBLER, E. LOMMATZSCH, op. cit., II, 1245 : bezaubern, berüchen.

32. PANNIER, op. cit., p. 51.

33. BERTELLI, art. cit., p. 103.

34. VinCENT DE BeAUVAIs, op. cit., t. I, lib. VIII, C XL (cf. n. 13).

35. Dictionnaire des Lettres françaises, Le Moyen Âge, Paris, 1964, p. 650.

36. BERTELLI, art. cit., p. 121. 
37. Encore au XIV ${ }^{\mathrm{e}}$ siècle Jean Corbechon insiste sur le sentiment de merveille suscité par l'observation des expériences sur l'aimant : Paris, BnF, ms. fr. 16993, fo 234b, lignes 12 sq. : (le lxij chapitre de l'aimant) : "C'est grant merveille comment l'aimant aime le fer autant, non pas tant seulement l'aimant le trait mais qui plus est, un fer trait l'autre quant il a touché a l'aimant sicomme il appert du coustel qui prant l'aguille quant il a touché a ceste pierre ». Il est aussi intéressant de noter que, par rapport à Barthélemy l'Anglais qui ne mentionne pas la boussole marine, la «traduction » de Corbechon ajoute l'aiguille aimantée.

38. Epistola Petri Peregrini de Maricourt Ad Sygerum de Foucaucourt Militem, dans P. PEREgRINUS DE MARICOURT, Opera, éd. Loris Sturlese et R. Thompson, Pise, 1995. On peut citer aussi, pour la traduction française et le commentaire, P. RADELET-DE GRAVE, et D. SPEISER, « Le "De magnete" de Pierre de Maricourt. Traduction et commentaire ", Revue d'histoire des sciences, 28, 1975, p. 193-234. En ce qui concerne la biographie et l'œuvre de Pierre de Maricourt, voir B. Boncompagni, «Sopra Pietro Peregrino di Maricourt e la sua epistola De magnete, Memoria Prima », Bullettino di bibliografia e di storia delle scienze matematiche e fisiche, 1, 1868, p. 1-32.

39. C. Segre, C. OsSOLA, op. cit., p. 63, 77-94.

40. [« Les savants narrent que l'aimant n'aurait le pouvoir d'attirer le fer que par le moyen de l'air. Aucune pierre, excepté celle de l'aimant, n'a autant de puissance d'attirer le fer, car n'en a pas le pouvoir. Ainsi, madame, Amour s'est rendu compte qu'il n'aurait pu m'attirer à soi que grâce à vous. En effet, il y a beaucoup de dames mais il n'y en a aucune vers laquelle Amour me fait tendre, à l'exception de vous, dame plaisante, qui abritez la force et le pouvoir »].

41. ["Et vous, soyez sûre que le pouvoir de votre beauté m'attire à vous, de la même façon que l'aimant attire l'aiguille par nature »]. ConTINI, Poeti del Duecento, MilanNaples, 1960 (La letteratura italiana. Storia e testi) vol. 2, t. I, p. 153 sq., 27-30.

42. N'oublions pas que la forme «close » par excellence, le sonnet, a été « inventée » par Giacomo da Lentini.

\section{RÉSUMÉS}

Cet article est une analyse de la première strophe de la chanson Tout autresi com l'aïmanz deçoit, de Gautier d'Épinal (xIII ${ }^{\mathrm{e}}$ siècle), qui décrit le charme de la dame par le biais d'une comparaison avec l'aimant. Le choix des mots, des expressions et des renvois à d'autres passages à l'intérieur de la même chanson témoigne du renouvellement de la lyrique du XIII ${ }^{\mathrm{e}}$ siècle dans ses figures au contact des savoirs scientifiques. En essayant de repérer les sources littéraires et scientifiques dans lesquelles Gautier aurait puisé et renouvelé son image, nous constatons que son apport trouve des correspondances très ponctuelles dans le milieu le plus expérimental de l'époque, celui de l'« École » poétique sicilienne de Frédéric II.

"Ä̈manz »: a Chapter of Gautier D'Épinal's «Lyrical Encyclopedia ». This article is an analysis of the first stanza of Tout autresi com l'aïmanz deçoit, a lyric by Gautier d'Épinal (13th century), which describes the appeal of the dame through a comparison with the magnet. The choice of words, 
expressions and items which recall other passages within the same song stand for the renewal of poetic imagery in the 13th century, which was brought about thanks to the contact with scientific knowledge. In trying to find the literary and scientific sources, which resulted in Gautier's imagery, we have also noted that his contribution has many analogies with what was going on in the most experimental cultural setting of his day, Frederic II's poetic Sicilian «School».

INDEX

Keywords : Gautier, Épinal, song, magnet, encyclopedia

Mots-clés : chanson, aimant, encyclopédie

\section{AUTEUR}

\section{GERMANA SCHIASSI}

Università di Bologna, Dipartimento di Filologia Romanza. Facoltà di Lingue e Letterature Straniere Moderne, via Cartoleria, 5, I-40 124 Bologna. 